\title{
Computer vision and deep learning automates nocturnal rainforest ant tracking to provide insight
}

4 Natalie Imirzian ${ }^{1 *}$, Yizhe Zhang ${ }^{2}$, Christoph Kurze ${ }^{1,3}$, Raquel G. Loreto ${ }^{1,4}$, Danny Z. Chen ${ }^{2}$, \& David P.

$$
\text { Hughes }{ }^{1,3^{*}}
$$

$6 \quad{ }^{1}$ Department of Entomology, Pennsylvania State University, University Park, PA, USA

$7 \quad{ }^{2}$ Department of Computer Science \& Engineering, University of Notre Dame, Notre Dame, IN, USA

$8 \quad{ }^{3}$ Department of Biology, Pennsylvania State University, University Park, PA, USA

$9{ }^{4}$ Department of Entomology, Federal University of Viçosa, Viçosa, Minas Gerais, Brazil

$10 *$ Corresponding authors: nsi2@psu.edu; dph14@psu.edu

\section{Abstract}

Determining how ant colonies optimize foraging while mitigating disease risk provides insight into how the ants have achieved ecological success. Fungal infected cadavers surround the main foraging trails of the carpenter ant Camponotus rufipes, offering a system to study how foragers behave given the persistent occurrence of disease threats. Studies on social insect foraging behavior typically require many hours of human labor due to the high density of individuals. To overcome this, we developed deep learning based computer vision algorithms to track foraging ants, frame-by-frame, from video footage. We found foragers can be divided into behavioral categories based on how straight they walk across the trail. Eighty percent of ants walk directly across the trail, while $20 \%$ wander or circle when crossing the trail. Departure from the main trail encourages exploration of new areas and could enhance discovery of new food resources. Conversely, results from our agent-based model simulations suggest deviation from a straight path exposes foragers to more infectious fungal spores. Consistency in walking behavior may

24 protect most ants from infection, while the foragers with increased exposure due to their mode of walking 25 could be a sufficient number of new hosts to sustain disease in this environment. 


\section{Introduction}

28 Resource acquisition drives animals into new territories, while threat avoidance limits where animals move. A consistent threat is the presence of infectious propagules of parasites and these are hypothesized to be major determinants of the distribution of animals in the wild ${ }^{1}$. Examples of animals avoiding pathogen contaminated areas span diverse taxa, from mammals to insects, implying anti-parasite behavior is widespread ${ }^{1-5}$. Central place foragers are interesting in the context of parasite avoidance as they must obtain food while avoiding threats with the additional constraint of returning to a defined location after each trip. For volant central place foragers, like wasps, bees, bats and birds, much of the trip is through the air likely reducing contact with infectious material. However, for taxa which walk on the ground (e.g. ants), encounters with parasite propagules are presumably higher ${ }^{6}$. To effectively study such pressure, it is crucial to use systems where we can study foragers in nature, surrounded by their naturally occurring pathogens.

The foraging strategies of ants range from workers searching and retrieving food entirely cases, these chemical trails develop into semi-permanent trails known as 'trunk trails ${ }^{9}$. Trunk trails stimulate research interest largely from the perspective of the self-organization behavior of ants, such as

43 how ants regulate traffic ${ }^{9-11}$. Trunk trails have also been studied from the perspective of their temporal

44 and spatial dynamics as well as their energetic value in terms of efforts expended and resources

45 obtained $^{12,13}$. Yet, studies have not investigated how utilizing the same trails day after day impacts the

46 exposure of ants to parasitism. Moreover, studies on ant foraging have largely occurred in a laboratory

47 setting, and of the work that took place in the field, most studies relied on human observation or

48 manipulated the environment in some way (see references in Supplementary Table S1). An ant species

49 that forages collectively and predictably in time and space would be useful to assess the relationship

50 between trail behavior and disease risk.

A potential system is the carpenter ant Camponotus rufipes in southeastern Brazil, which forms

52 trunk trails lasting for multiple months ${ }^{14,15}$. Colonies of this ant were recorded as having a chronic 
53 infection by the fungal parasite Ophiocordyceps camponoti-rufipides across 20 months ${ }^{16,17}$. This fungus

54 manipulates foragers to leave the nest and die biting the underside of a leaf ${ }^{17,18}$. To complete its lifecycle,

55 the fungus must grow out of the ant cadaver and form a fruiting body that releases spores onto the ground

56 below that will infect other ants ${ }^{18}$. Cadavers are found attached to leaves surrounding the ant nest ${ }^{17}$. The

57 chronic nature of infection at the colony level means the spores of the pathogen are continuously in the

58 environment from the perspective of the foragers. The spores are curved and large $\left(80-95\right.$ microns $\left.^{16}\right)$

59 implying they do not travel far and land on the nearby trails once released from ant cadavers that hang

60 above trails. Spores germinate to produce infectious secondary spores on hairs (capilliconidia) which

61 attach to ants as they walk over them ${ }^{19}$. Thus, infection does not require a spore to hit an ant as it walks on

62 a trail below a cadaver. Instead, the trail substrate itself serves as the source of contamination.

Foragers of the carpenter ant $C$. rufipes mostly collect nectar from hemipteran secretions and

64 extrafloral sources ${ }^{14,20}$. The exploitation of a stable resource suggests that all foragers will emerge to walk

65 directly to the food source, utilizing trails near the colony entrance as a highway ${ }^{15}$. Evidence from other

66 systems demonstrate trunk trails as well organized for traffic flow ${ }^{10}$. Traffic is bi-directional on the trunk

67 trails of C. rufipes. Thus, we expect an even mixture of inbound and outbound ants as this is hypothesized

68 to increase flow ${ }^{21}$. If colonies can regulate the number of foragers on the trail to create a steady flow, we

69 expect forager speed to remain approximately constant throughout the foraging period as foragers are not

70 limited by the density of ants on the trail. Lastly, we are interested to see how the individual walking

71 behavior observed influences the likelihood of an ant encountering an infectious spore.

72 We set out to study trails of seven C. rufipes colonies in their undisturbed rainforest habitat with

73 both food sources and pathogens occurring at natural levels. We devised a system of recording trails using

74 infrared lights and modified cameras to contend with the nocturnal foraging of this species. To overcome

75 observer bias and ensure a larger body of data from which patterns may emerge, we used machine

76 learning to automate ant tracking. This provided us with a powerful dataset from which the movement

77 pattern of ants throughout a foraging period can be examined. We then characterized the forager

78 trajectories on speed, straightness, and direction. Based on these measurements, we were able to classify 
79 ants on the trail into behavioral groups. Using an agent-based model based on our data, we suggest a

80 mechanism for the maintenance of disease in this system.

82 Methods

83 Study site

84 Fieldwork took place at the Research Station of Mata do Paraíso, Universidade Federal de Viçosa, Minas

85 Gerais, Southeast Brazil (2048’08 S 4252’31 W) between 10 and 25 January 2017. The carpenter ant

86 Camponotus rufipes is abundant in this area, forming trails lasting multiple months ${ }^{14,15}$. Trails of $C$.

87 rufipes are typically found on 'bridges' composed of woody debris, lianas and tree branches and are

88 rarely directly on the forest floor ${ }^{15}$. Ants forage at night and activity peaks in the early evening ${ }^{15}$.

Trail filming

91 Trails from seven different C. rufipes nests were filmed between 10 and 25 January 2017. Nests were

92 selected based on their location and structure. Only nests found above the ground with nest material

93 clearly visible were used. Trails were filmed before a branching point from the main trail so that ants

94 were filmed coming directly from or towards the nest. In the case where multiple trails came from one

95 nest, the busiest trails were selected. The width of the branches filmed ranged from $0.8 \mathrm{~cm}$ to $7 \mathrm{~cm}$ (mean

$96 \pm$ standard deviation; $2.97 \mathrm{~cm} \pm 2.53$ ) and the length of the area filmed for all branches was

97 approximately $15 \mathrm{~cm}$.

98 GoPro cameras (model: HERO 3+, GoPro, Inc., San Mateo, USA) with a modified infrared filter

99 (RageCams.com, Michigan, USA) were used for filming. Stakes were placed 30 centimeters from the

100 trails and $30 \mathrm{~cm}$ medium trigger clamps (DWHT83140, DeWalt, Towson, USA) were attached to the

101 stakes. Cameras were attached to clamps so that cameras were approximately 30 centimeters above the

102 trails looking down at the ants walking on the trails (Supplementary Fig. S1). An additional camera was

103 placed on the stake, looking sideways at the ants, to allow another perspective for behavioral analysis.

104 Filming lasted from 19:30 to 00:00 for 4-7 nights for each trail (Supplementary Table S2). Timing of 
filming was based on previous work showing activity begins around 19:30 and peaks around 21:0015.

UniPower, São Paulo, Brazil) to allow illumination of the trail without disturbing the behavior of the ants.

108 The camera batteries lasted for approximately 1.5 hours, so the battery was changed once in the middle of

109 a filming period. Slight adjustments in where the trail was positioned in the video view would sometimes

110 occur at this time. Figure 1a shows an example image of a trail filmed and images of the remaining trails

111 filmed are found in Supplementary Figure S2.

Automated ant tracking

114 A total of 78 hours and 56 minutes of video were recorded for seven colonies across four nights

115 (Supplementary Table S2). We developed a machine learning approach to process and analyze these

116 videos using a deep learning based segmentation model that identified ants as they came onto the screen

117 and tracked them as they moved across the screen.

Our automatic ant tracking method contains two main processes: (1) detecting ants in each image

119 frame of all videos, and (2) building ant trajectories for every video based on the detected ants.

120 Commonly, deep learning schemes require a large amount of labeled ground truth data for model training.

121 Since our dataset is quite large ( $>8$ million image frames), we aimed to generate sufficient labeled data

122 for training our deep learning model without incurring excessive human labeling effort. Also due to the

123 large size of our dataset, common active learning based sample selection methods (e.g. ${ }^{22}$ ) are not efficient.

124 The goal of ant detection is to build ant movement trajectories and since ant trajectories normally span

125 multiple consecutive frames in videos, detected ant positions in earlier frames assist with ant detection in

126 later consecutive frames. That is, while ant detection forms a basis for building ant trajectories,

127 trajectories of detected ants may also help ant detection. Hence, we designed our trajectory building

128 procedure such that it not only can track detected ants but also can provide cues to indicate where (which

129 frames and locations) there might be inconsistencies in ant trajectories and difficult scenarios for ant

130 detection (e.g. densely clustered ants). We used such cues to select difficult cases from the frames for 
131 labeling to improve the deep learning detection model as well as the ant detection results. Therefore, our

132 detection-tracking method consists of two rounds (with the second round improving the detection and

133 tracking results of the first round), and each round performs two major steps, ant detection and trajectory

134 building, as described below.

136 (1) Ant detection. This aims to detect ants in all the frames of the videos. We applied a novel object

137 detection and segmentation model, Mask R-CNN ${ }^{23}$, to automatically detect ants in every frame.

(2) Ant trajectory building. Given the detected ants in each frame, the next step is to form ant trajectories

140 that connect detected ants frame-by-frame in videos. We formulated this ant trajectory building problem

141 as a transportation problem, that is, between every two consecutive frames in each video, we find an

142 optimal transportation (for ants) that corresponds to real movement of ants. In this transportation

143 formulation, each detected ant in frame $K$ can be viewed as a 'supplier' and each detected ant in frame

$144 K+1$ can be viewed as a 'receiver'. The dissimilarity (based on spatial distance and appearance

145 difference) between ants in two consecutive frames is a measure of how much 'cost' it would take to

146 transport (move) one ant in frame $K$ to another in frame $K+1$. The objective is to transport detected ants

147 (as many as possible) in frame $K$ to frame $K+1$ with the minimum total cost. Optimal transportation based

148 tracking methods are known to be effective for tracking sets of moving and changing objects in image

149 sequences ${ }^{24,25}$.

151 In the first round, we randomly selected frames to label as training data. This allowed us to quickly and

152 unbiasedly obtain data samples for training a decent detection model. We then applied the trained model

153 to all of the frames to produce ant detection results. Next, we conducted trajectory building on detected

154 ants to form the ant trajectories. Besides tracking ant movement, our trajectory building procedure in the

155 first round also provided cues for identifying inconsistencies in ant trajectories and difficult cases in the

156 frames for ant detection. In the second round, we applied training data selection to those difficult cases to 
157 find additional frames for labeling, and the enlarged training dataset thus obtained was used to re-train the

158 Mask R-CNN detection model. The re-trained detection model was then applied to all the frames to

159 produce the final ant detection results, which were used to build the final ant trajectories in the videos.

160 To identify difficult cases for additional training data selection, we used the following set of

161 measures to capture possible errors in ant detection and trajectory results. (i) Ant speed: At a place where

162 ants usually do not move very fast but a fast movement is suggested by the optimal transportation

163 solution, this instance might indicate an error in ant detection. (ii) Missing ants in the middle part of a tree

164 branch: When the optimal transportation solution does not find a corresponding ant instance in the next

165 frame in the interior section of a tree branch, it might suggest a missing data point in ant detection. (iii)

166 Ant identification (ID) switching: Each detected ant was assigned an ID number; when multiple ants are

167 seen at spatially close interaction and slight changes on the dissimilarity scores among these ants give

168 largely different solutions for the optimal transportation problem, this might suggest an ant ID switch

169 error. Based on these observations and measures, our trajectory building process can help identify

170 difficult detection and tracking cases for additional training data selection to improve model performance.

171 Overall, our automatic ant detection and tracking method extracted the $x$ and $y$ coordinates in

172 pixels of detected ants in every frame and assigned each ant an identification number (Fig. 1a;

173 Supplementary Video S1). Ant identification numbers were used to form ant trajectories used in further

174 analysis.

\section{Error assessment}

177 To assess the accuracy of the computer model, we watched a subset of videos and determined the error

178 rate. GoPro cameras automatically divide footage into 26-minute-long videos, so one night of footage at a

179 single trail has 6 to 10 videos. This provides a way of checking the accuracy of the computer tracking at

180 random points throughout a night. We first error checked videos from the middle of the night (when the

181 trails should be busiest) to determine if the data from that colony was high enough quality to use in our

182 analysis. If the error rate was sufficiently low, we continued to error check all videos and nights for that 
colony. To error check, we counted the number of ant trajectories with errors out of the first 15-30 tracked

ants. The number of ant trajectories checked varied because videos from early in the foraging period sometimes had fewer ants.

To ensure consistency in the type of ant trajectories that were analyzed, trajectories beginning in the middle of the field of video view were removed. This created uniformity between all colonies and nights in the type of ants that were compared as it focused on the ants that made it from one end of the trail to the other completely in the view of the video.

\section{Trajectory analysis}

192 We used R version 3.4.4 and RStudio version 1.1.447 for all analyses ${ }^{26,27}$. Ant location data was frameby-frame, so we used the native frame rate of the cameras (29.97 or 25 frames per second; the default setting of the cameras varied) to convert the time in frames to seconds and then used the start times of each video to convert it to real time (Supplementary Table S2). To convert ant location data from pixels to centimeters, we placed a ruler in each video to determine the conversion factor (Supplementary Fig. 2). speed, overall direction, time on the trail, and straightness. Average speed was taken as the total distance an ant travels while in the video over the time it takes for them to travel that distance. Overall direction was whether the ant headed away from or towards the nest which we determined based on where the ant

201 entered and exited the video view. A variety of measures are used to determine the straightness or

202 tortuosity of an animal's movement path ${ }^{28,29}$. Ant movement on trunk trails is expected to move in an 203 oriented direction, and not be a random search path, thus we used the simplest measure, the straightness 204 index ${ }^{29}$. The straightness index (ST) is a ratio between the net displacement and total path length:

$$
\mathrm{ST}=\mathrm{d} / \mathrm{L}
$$


210 To assess the influence of foraging style on disease risk, we developed an agent-based model in NetLogo

$2116.0 .2^{30}$ based on the walking style of the ants in our videos (full details in Supplementary Materials). This

212 model tested how walking straight influences the hypothetical number of spores an ant picks up. Spore

213 density varied from $10 \%$ to $100 \%$ of the patches in the environment covered in spores. We varied spore

214 density in $10 \%$ increments, leading to 10 different spore density conditions. The straightness of an ant

215 varied from 0 to 1 in 0.01 increments, leading to 101 different straightness scores. The model was run 30

216 times for each combination of parameters (1010 total combinations) leading to a total of 30300 runs.

218 Statistical analysis

219 A linear mixed-effects models fit was used to assess whether the speed of ant changes over a foraging

220 period. The model was generated using the lmer function in the R package' 1 me $4{ }^{31}$, with speed as the

221 fixed effect and colony and date as the random effects. The package 'ImerTest' ${ }^{32}$ was used to generate p-

222 values. We checked the plotted residuals to ensure homoscedasticity prior to utilizing the results of the

223 model. We used linear regression to analyze the results of the agent-based model, with the straightness

224 value as the predictor of proportion of spores picked up in the environment with a log transformation to

225 control for skew.

226

\section{Data Availability}

228 The original videos and data analyzed in this study will be accessible through ScholarSphere

229 (https://scholarsphere.psu.edu/) upon publication of this study. 


\section{Results}

Automated tracking performance

237 The automated tracking of ants in video frames resulted in 20,230,585 data points on ant movement. The

238 model had two types of accuracy against which it can be judged, relative to a human. The first is species

239 accuracy (detection accuracy) which is a measure of how well the model recognized the correct species of

240 ant. The model correctly detected C. rufipes ants with an accuracy of $97.86 \%$. The model picked up other

241 insects or species of ants on the trail (false positive) or failed to detect a C. rufipes ant as it went across

242 the trail $2.14 \%$ of the time.

The second accuracy measurement is tracking accuracy. The computer had to detect C. rufipes

244 ants and follow them as they moved across the screen. If an ant moved in a straight line this required the

245 computer to recognize and track that ant for about 4 seconds or 120 frames. The computer assigned

246 identification numbers to individual ants to follow an ant as it travelled across the screen. The machine

247 learning model sometimes made errors in doing this. The computer may switch identification numbers

248 when ants walked too closely together (Supplementary Video S2). The average tracking accuracy for all

249 colonies was 78.70\%. The tracking accuracy was the lowest for MP2 (40.0\%), MP11 (31.7\%), and MP17

250 (50.6\%). Identification number switches commonly happened in colonies MP2 and MP11. These trails

251 were very thin and introduced more challenges in determining the trajectories of individual ants, so they

252 were removed from further analysis. We have additionally removed MP17 as an obstruction in the trail

253 led to ants departing from the branch and walking underneath leaves (Supplementary Video S3). Ants

254 disappearing under leaf debris made it difficult to track an individual ant. We have made all videos and

255 data available as we expect improved future machine learning models can make use of them.

256 The exclusion of these colonies brought the size of the dataset to 8,505,784 data points on ant

257 movement from four colonies: MP1, MP6, MP10, and MP16. The large reduction of the number of data

258 points from the elimination of 3 colonies can be attributed to the errors in these branches, where the

259 density of individuals in congested areas lead to a false inflation of the number of ants and overall data

260 points. The data points from the 4 included colonies represents the movement data for 64,499 ants. The 
average tracking accuracy of the remaining colonies was 81.39\% (MP1: 72.0\%; MP6: 82.1\%; MP10: 77.2\%; MP16: 92.1\%). Most errors were due to an identification number switching to a different ant (8.28\%). The high error rate for MP1 could be attributed to the darkness of the videos causing the model to miss part of an ant's trajectory or failing to detect an ant in the dark areas of the trail. If we consider only the errors where a number is on a wrong ant or a number is not on an ant, the accuracy improves greatly (overall: 90.94\%; MP1: 91.5\%; MP6: 88.8\%; MP10: 86.6\%; MP16: 96.3\%). We are mainly concerned with the direction and shape of trajectories, and the main error that impacts an individual ant's trajectory is when ants switch to the wrong identification number, so the second calculation of accuracy rate is more reflective of this.

\section{Collective movement pattern}

272 Most ants walk on the same area of the available trail space (Fig. 1). Ants often follow each other,

273 walking across the same area (Supplementary Video S4). The trail usage pattern is consistent between

274 nights (Fig. 1c). The mean speed of all ants from all colonies and nights was $5.19 \mathrm{~cm} / \mathrm{s} \pm 1.61$ (standard

275 deviation). The average speed of the colonies ranged from $4.74 \mathrm{~cm} / \mathrm{s}$ to $5.62 \mathrm{~cm} / \mathrm{s}$ and within colony

276 variability in speed was similar between colonies (mean $(\mathrm{cm} / \mathrm{s}) \pm$ standard deviation; MP1: 4.99 \pm 1.69 ;

277 MP6: 5.62 \pm 1.60 ; MP10: 4.88 \pm 1.53 ; MP16: 4.74 \pm 1.41$)$. The results of the linear mixed effects model

278 showed that ant speed decreases by $0.50 \mathrm{~cm} / \mathrm{s} \pm 0.07$ (standard error) throughout the night $\left(\mathrm{t}_{(96.45)}=-7.12\right.$, $279 \mathrm{p}<0.0001)$ (Supplementary Fig. S3).

\section{Individual movement pattern}

282 Although most ants walked on the same area of the branch (Fig. 1b-c), there was a subset of ants that 283 walked differently based on the straightness score (Fig. 2). Based on the behavioral analysis of videos, 284 ants that had a straightness score of close to one walked straight across the trail as was expected (Fig. 2b; 285 Supplementary Video S5). We found that $80.8 \%$ of ants had a straightness score from 0.75 to $1(\mathrm{n}=$ 
50,813). We labelled these ants as 'direct walkers'. Ants with an intermediate straightness score typically

made it from one end of the trail to the other, but spent time wandering and covering more area of the trail

(Fig. 2b; Supplementary Video S5). We labelled these ants as 'wanderers'. They represent $13.0 \%$ of ants and had a straightness score from 0.25 to $0.75(n=8,194)$. By contrast, $6.2 \%$ of ants had a straightness score of less than $0.25(n=3,869)$. These ants with a very low straightness score typically circled on the trail consequently entering and exiting on the same side of the video view (Fig. 2c; Supplementary Video S5). We labelled these ants as 'circlers'.

The wanderers and circlers constituted the minority of records (13\% and $6.2 \%$ respectively). We observed these two behavioral phenotypes regardless of whether there were other ants in the area (Supplementary Video S5). These ants often stopped and groomed or antennated the trail or air (Supplementary Video S6). However, direct walkers were also observed stopping and grooming their antennae (Supplementary Video S7). There was a significant effect of straightness group on time spent on the trail for all three groups (Fig. 3e; one-way ANOVA; $\mathrm{F}_{(2,64495)}=14350, \mathrm{p}<0.0001$ ). Post hoc comparisons using the Tukey Test indicates circlers did not spend more time on the trail (mean=8.1 seconds, $\mathrm{SD}=6.85$ ) than wanderers (mean=7.58 seconds, $\mathrm{SD}=3.59$ ), but both spent significantly more time on the trail than direct walkers (mean=3.88 seconds, $\mathrm{SD}=1.42$ ).

\section{Temporal movement pattern}

304 The flow of all three groups of ants (direct walkers/wanderers/circlers) in and out of the nest was 305 approximately the same throughout the night (Fig. 3). There is a large increase in the number of direct

306 walkers on the trail throughout the night, while the number of wanderers and circlers throughout the night 307 is relatively constant.

309 Agent-based model

310 Based on the results obtained from our agent based model, walking straight significantly decreases the 311 proportion of potential spores an ant picks up in an environment (linear regression: $\mathrm{F}_{(1,30298)}=5,458, \mathrm{p}<$ 
0.0001; Fig. 4a). The three different groups of foragers differed in the number of spores they pick up in

313 the environment regardless of spore density $\left(\mathrm{F}_{(2,30297)}=21,208, \mathrm{p}<0.0001\right.$; Fig. $\left.4 \mathrm{~b}\right)$. Circlers pick up

314 significantly more spores than wanderers and wanderers may pick up significantly more spores than direct

315 walkers (Tukey Test; $\mathrm{p}<0.0001)$.

\section{Discussion}

318 Our study utilized an unobtrusive filming set-up to record behavioral data on more than 64,000 ants

319 moving in a rainforest at night in an area of high disease pressure. The study design facilitated the capture

320 of natural ant behavior unaffected by either a laboratory environment or proximity to human observers.

321 Combining this approach with computer vision techniques increases the scale at which we can study

322 animal behavior. Using computer vision and deep learning we collected approximately 20 million ant

323 movement data points from 80 hours of nighttime video. A previous study, using humans to score the

324 positions of ants in each frame, required approximately 1,600 hours of human work to create a dataset of

3256.9 million data points (Modlmeier et al., in review). Advances in camera technology improving our

326 nighttime recording capabilities along with increased computing power allowing machine learning to

327 identify individuals promotes research on natural animal behavior.

328 For our study on ant behavior in the context of disease transmission, the scale of this data

329 detected higher level patterns likely unobservable with a less detailed dataset. Our data shows ants

330 flowing in and out of the nest at approximately the same rate (Fig. 3). Work on harvester ants

331 (Pogonomyrmex barbatus) has shown that the feedback from returning foragers stimulates inactive

332 foragers to leave on a new trip ${ }^{33}$. Our even flow rate also validates work on Argentine ants (Linepithma

333 humile) showing ants exiting and entering the nest at approximately the same rate in the summer ${ }^{34}$. Ant

334 colonies operate through local interactions and without centralized control, so there is no authority

335 controlling when ants leave and return to the nest $\mathrm{t}^{35,36}$. The lack of centralized control combined with the

336 even flow rate gives insight into the processes occurring within a nest, with returning foragers likely

337 stimulating new foragers to leave the nest. 
Given that there is no centralized plan for foraging, it is impressive that the same foraging pattern

arises on different days (Fig. 1c). This consistent trail usage pattern, along with most ants walking straight

across the trail (Fig. 2a) likely emerges from the use of a chemical trail, which this species of ant $(C$.

341 rufipes) is known to use ${ }^{37}$. For ants to walk on the same area of the trail on different nights, the trail

342 pheromone must either persist between foraging periods or foragers repeatedly reinforce the fastest route

343 across the branch each night. While rare, we observed some ants on these trails during the daytime, and

344 other studies have observed C. rufipes foraging during the day ${ }^{20}$. This could allow the trail to be

345 reinforced around the clock. Alternatively, laboratory studies have demonstrated ants as preferentially

346 selecting the shortest route to food ${ }^{38,39}$. The path that receives more pheromone will be reinforced

347 quicker ${ }^{40}$. Thus, each night the portion of the trail that ants walk on fastest could reach a higher

348 concentration of trail pheromone quicker, leading to the pattern observed.

The texture of the tree branch could also drive the space usage pattern, as substrate and landscape

that $C$. rufipes foragers in this environment prefer to walk on woody debris because they walk faster on

this material than on the forest floor (see Supplementary Video S8 for an example of how ants are

investigation of the straightness index of the ants. As expected, the straightness index of most ants was

357 prefer on the path that deviates the least from their original direction of $\operatorname{travel}^{43}$. Straighter individual

358 paths enhance information spread and increase the chance that an ant will find food ${ }^{44,45}$, perhaps making

359 this pattern beneficial to the collective colony in resource acquisition.

Despite the dominance of ants walking straight across the trail, a proportion of foragers wandered

361 before making it across the trail and another group circled the trail and headed back in their original

362 direction (Supplementary Video S5). Other species of ants provide evidence of different roles within

363 foragers, such as patrolling, trail maintenance, and defense. Patrollers in harvester ants are the first to 
leave the nest in a foraging period and determine which trails the colony will use that day ${ }^{46}$. However, if the subtypes in this study were patrollers, we would expect there to be more of them at the beginning of the night which is not the case (Fig. 3). The leaf cutting ant Atta cepahlotes, which also forms consistent trails, has a special class of foragers involved in trail maintenance ${ }^{13,47}$. Ants were observed carrying leaves (Supplementary Video S9), although this could be for nest material and not trail cleaning. Another role could be maintaining the pheromone trail. For example, Atta sexdens minims help with the pheromone trail instead of food transport ${ }^{47}$. Ants were observed dragging their gaster on the trail likely depositing trail pheromone (Supplementary Video S10). However, it seems unlikely wanderers and circlers were involved in maintaining the pheromone trail, as they should walk straight across the trail to ensure the pheromone trail was on the most direct path. trail (Fig. 2c) and were observed stopping and antennating (Supplementary Video S8). Smaller workers

377 serves as a defense against parasitoid Phorid flies ${ }^{48}$. Flies, that could possibly be parasitoids, were observed closely following ants on the trail and in some cases appearing to land which may indicate laying eggs on the ants which later become endoparasitoids (Supplementary Video S11) although the prevalence of parasitoid flies attacking C. rufipes is unknown. We have observed adult ants infected by decapitating phorid flies in our study area (Supplementary Video S12). colony and instead demonstrate differences in response plasticity, as individuals may differ in their

384 detection of the pheromone trail. Bumblebees (Bombus terrestris) vary in their antennal sensitivity to 385 odors and different behavioral thresholds have been found for castes of the ant Pheidole pallidula ${ }^{49,50}$.

386 Heterogeneity can be beneficial to the collective colony in tasks such as selecting a new nest ${ }^{51}$. In the 387 context of trunk trail foraging, it could encourage exploration and increase the chance of discovery of 388 high value food items. Camponotus rufipes typically uses trunk trails to exploit dependable food 389 resources such as hemipteran secretions or extrafloral secretions. If the colony always remains on the 
trunk trails, they might deplete their dependable source of food and not have a suitable alternative.

391 Argentine ants are able to adapt trails in response to resource availability ${ }^{52}$. We suggest wandering and 392 circling as a mechanism for C. rufipes ants to similarly respond to changes in resource availability. We

393 filmed only a small area of the foraging trails, providing a brief snapshot of an ant's behavior. To know

394 whether wanderers or circlers are more likely to wander from the trail and discover new food resources, 395 one would need to follow individual ants during an entire foraging trip, which was beyond the scope of 396 this study. groups represent fixed behavioral groups or if they just demonstrate variation in individual behavior over time. Campos et al. (2016) studied the activity patterns of Aphaenogaster sensilis ants and found foraging trajectories to be descriptively similar with individual temporal activity patterns showing greater variation. In the context of our study, perhaps all ants engage in wandering or circling behavior on these trails, and it is related to their temporal activity pattern and not their behavioral role. could impact the maintenance of disease in this environment. Fungal infected cadavers surround these trunk trails, likely dropping spores directly onto the trails below ${ }^{17}$. It is not possible to quantify the abundance and distribution of micron sized spores on trails in a forest, but the long term tracking of cadaver abundance and the proximity to the trails implies spore presence on the foraging trails ${ }^{17}$. According to our simulations, walking in a straight line reduces a forager's risk of picking up spores (Fig.

411 occur as the circlers and wanderers deviate from the straight path increasing their probability of picking 412 up spores and maintaining a chronic infection of the colony.

413 If the risk of infection is larger for the circlers and wanderers (Fig. 4), why do these subgroups

414 still exist? Social insects have members of the colony known as scouts that assist in discovering and 415 recruiting the colony to new food sources ${ }^{54-57}$. The wandering and circling behavior observed in this study 
could reflect the individual ant's role in food discovery, instead of food retrieval. Colonies with this

417 variability in forager behavior are perhaps better able to obtain resources, improving their reproductive

418 success and maintaining the diversity in forager behavior. Simultaneously, it allows persistence of the

419 fungal parasite in the system, but the loss these ants is likely not enough to have a selective impact on the

420 colony, as it is only a small percentage of foragers as suggested by Loreto et al. (2014). In the harvester

421 ant Pogonomyrmex owyheei, less than 10\% of the colony foraged and it was usually less than $6 \%$ at one

422 time ${ }^{58}$. Reproductive success was also hardly impacted when harvester ant foragers were restricted from

423 foraging ${ }^{59}$ implying colonies usually gather more than enough food and fitness would likely not take a

424 huge hit from the loss of a few foragers. Scharf et al (2017) additionally demonstrated that colony fitness

425 (as measured through the number of reproductive individuals produced) remained unchanged from

426 parasitic infection. In our system, relatively few foragers appear to be infected and killed by the parasite ${ }^{17}$.

427 In addition, the density of spores in the trails could be very low, since the trail network occupies less than

$4282 \%$ of the nest surrounding area ${ }^{15}$. Understanding the direct relationship between ant cadavers (from

429 where spores are shot) and the trails (where new hosts are found) would improve our agent-based model

430 predictions and help to understand the importance of wanderers and circlers for colony disease risk

431 management.

432 Although these behavioral subtypes are only a small proportion of the colony, that small

433 proportion could be more than enough ants to sustain a parasitic fungus. Understanding how variation in

434 behavior influences pathogen risk provides information on the factors that shape the distribution of

435 animals in time and space. Computational techniques serve as a way to collect large datasets on animal

436 behavior, where one can begin to unearth the complex interactions between an animal and its habitat. 


\section{Figures}

a

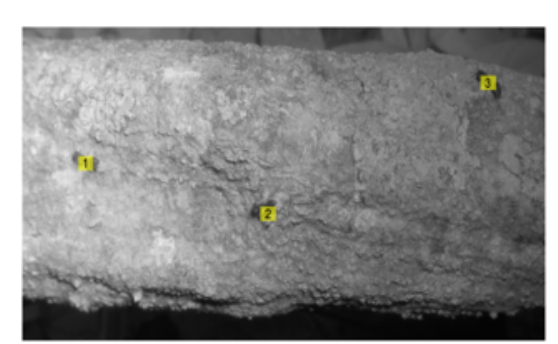

b

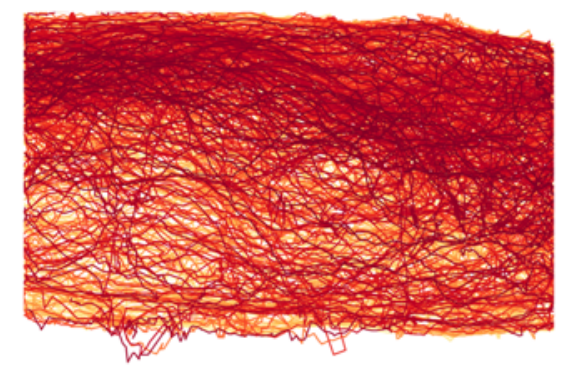

c

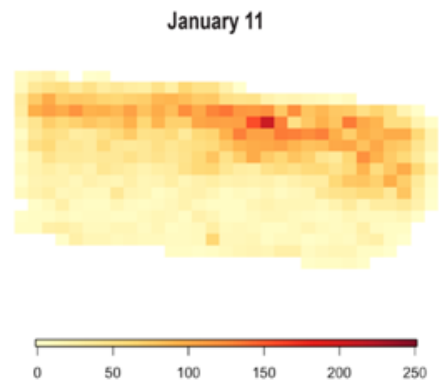

January 13

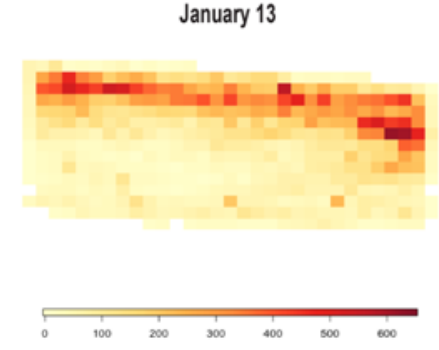

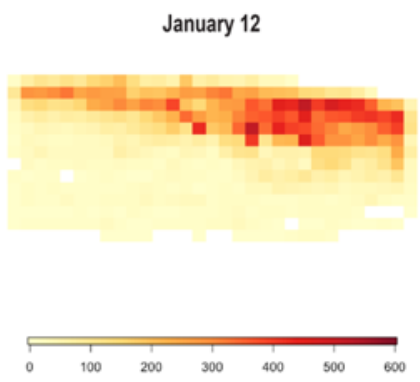

January 14

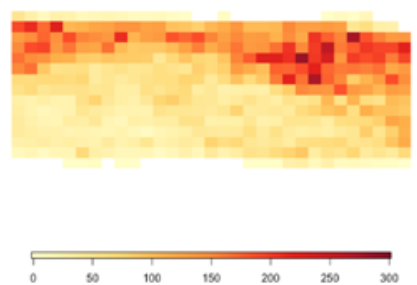

442 Figure 1. Trail image, trajectory overlay, and collective movement pattern. (a) Example image of a

443 trail filmed taken from GoPro footage from colony MP1. Ants are labeled with identification numbers.

444 (b) All of the trajectories from a single night of footage (January 14) at colony MP1. Each line across the

445 trail represents a different ant, with the different colors distinguishing between different ant tracks. (c)

446 The trail space from (a) was divided into a grid with each square representing approximately $1 \mathrm{~cm}^{2}$. The

447 number of times an ant walks into a square of the grid was calculated and the darker colors represent

448 areas of the trail that ants walked over more. Each heatmap represents a different date (January 11

449 through January 14) from approximately the middle of the night to control for differences in the timing of

450 filming. Different scales were used for each night, due to variance in the number of ants that walked

451 across the trail. 


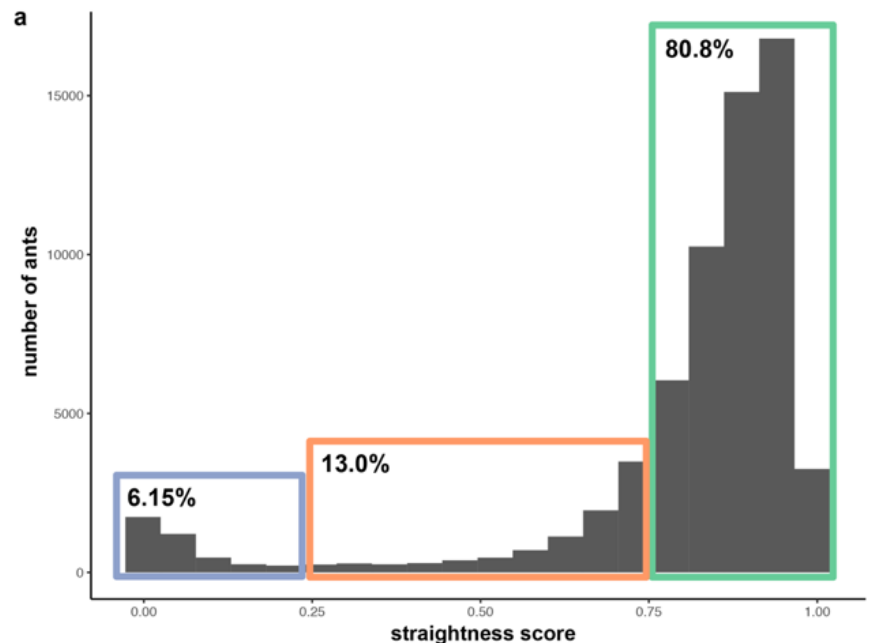

b
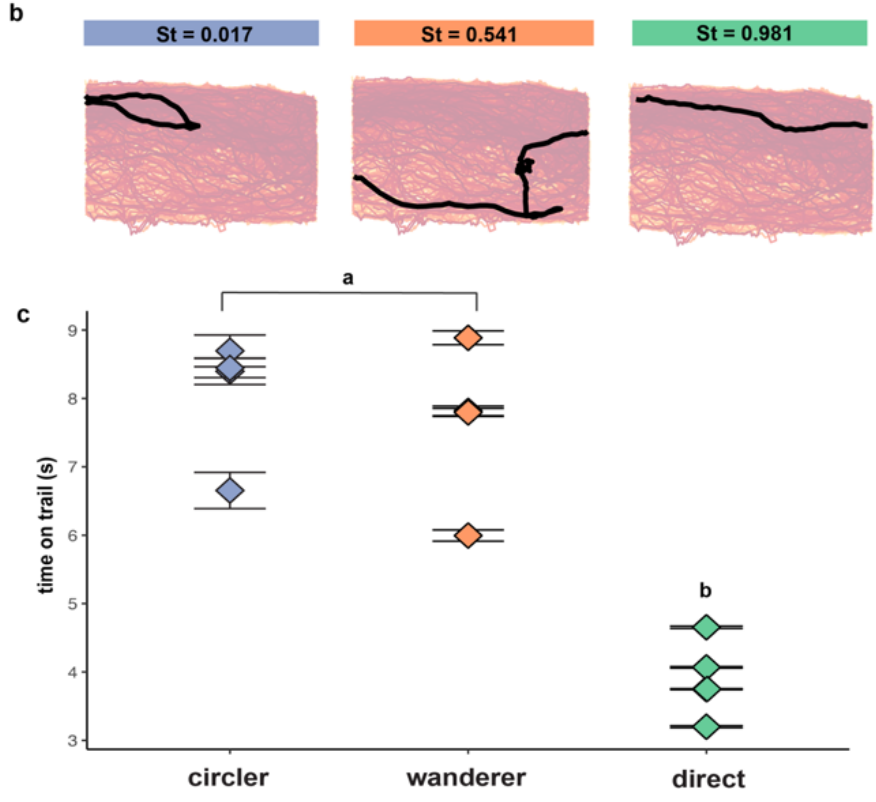

Figure 2. Different behavioral groups based on straightness score. (a) Histogram showing the distribution of straightness scores for all nights and colonies. (b) Example trajectories for a circler, wanderer and direct walker highlighted over all of the trajectories shown in Figure 1b. The straightness score (St) for that trajectory is included above. (c) Mean time spent moving across the trail in seconds for

459 each different behavioral group and colony \pm standard error of the mean. Different points within a

460 behavioral group represent different colonies. Superscripts indicate groups as significantly different $(p<$ $4610.0001)$ 


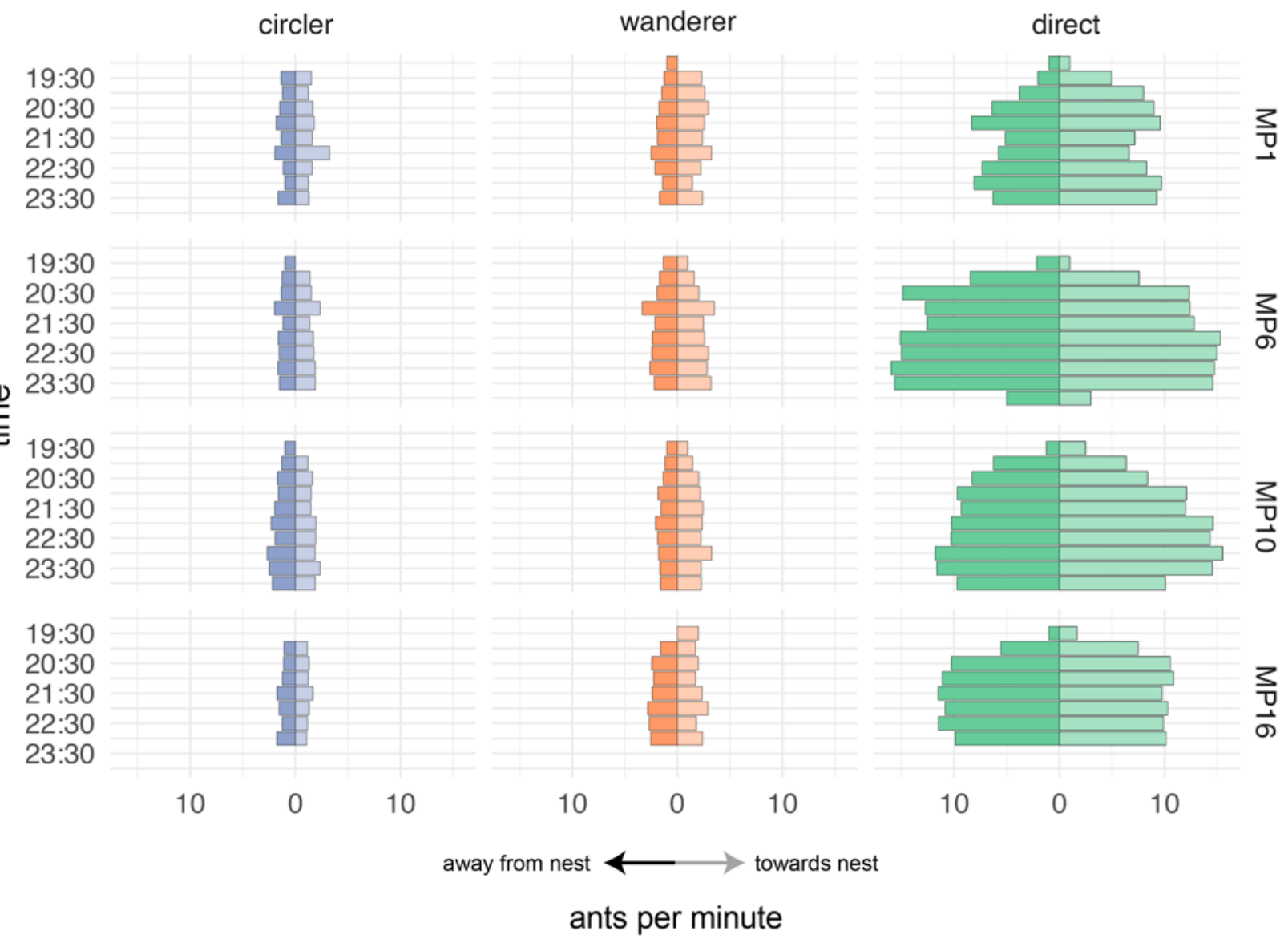

467 Figure 3. Distribution of different behavioral groups over time. Mean number of ants per minute in

468 each behavioral group in a 30-minute period going either away from the nest or towards to the nest.

469 Averaged across all nights for each colony. Right side numbers represent different colonies. 

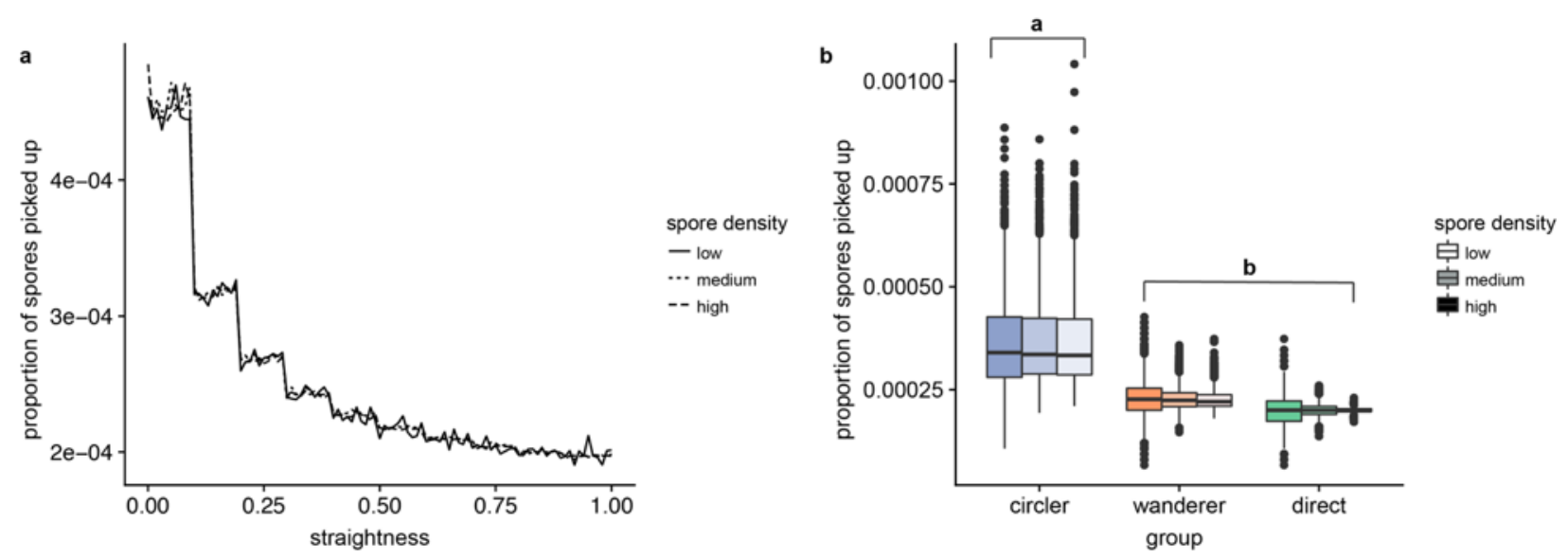
straightness scores (b) Data from (a), with straightness divided into behavioral groups.

485 Superscripts indicate groups as significantly different $(p<0.001)$. 


\section{References}

495 1. Moore, J. Parasites and the behavior of animals. (Oxford Univ. Press, 2002).

496 2. Fouks, B. \& Lattorff, H. M. G. Recognition and Avoidance of Contaminated Flowers by Foraging

497 Bumblebees (Bombus terrestris). PLOS ONE 6, e26328 (2011).

498 3. Weinstein, S. B., Moura, C. W., Mendez, J. F. \& Lafferty, K. D. Fear of feces? Tradeoffs between

499 disease risk and foraging drive animal activity around raccoon latrines. Oikos n/a-n/a (2018).

$500 \quad$ doi: $10.1111 /$ oik.04866

501 4. Villani, M. G. et al. Use of Radiography and Tunnel Castings for Observing Mole Cricket

502 (Orthoptera: Gryllotalpidae) Behavior in Soil. Am. Entomol. 48, 42-50 (2002).

503 5. Wynne, R., Morris, A. \& Rae, R. Behavioural avoidance by slugs and snails of the parasitic nematode Phasmarhabditis hermaphrodita. Biocontrol Sci. Technol. 26, 1129-1138 (2016).

6. Boomsma, J., Schmid-Hempel, P. \& Hughes, W. Life histories and parasite pressure across the major groups of social insects. in Insect evolutionary ecology: proceedings of the Royal Entomological Society’s 22nd Symposium 139-175 (CABI Publishing, 2005).

7. Hölldobler, B. \& Wilson, E. O. The ants. (Springer Verlag, 1990).

8. Edelstein-Keshet, L., Watmough, J. \& Ermentrout, G. B. Trail following in ants: individual properties determine population behaviour. Behav. Ecol. Sociobiol. 36, 119-133 (1995).

9. Couzin, I. D. \& Franks, N. R. Self-organized lane formation and optimized traffic flow in army ants. Proc. R. Soc. Lond. B Biol. Sci. 270, 139-146 (2003). (2010).

515 11. Edelstein-Keshet, L. Simple models for trail-following behaviour; Trunk trails versus individual $516 \quad$ foragers. J. Math. Biol. 32, 303-328 (1994).

517 12. Kost, C., Oliveira, E. G. de, Knoch, T. A. \& Wirth, R. Spatio-temporal permanence and plasticity of 518 foraging trails in young and mature leaf-cutting ant colonies (Atta spp.). J. Trop. Ecol. 21, 677-688 $519 \quad$ (2005). 
520 13. Howard, J. J. Costs of trail construction and maintenance in the leaf-cutting ant Atta columbica.

521 Behav. Ecol. Sociobiol. 49, 348-356 (2001).

522 14. Jaffe, K. \& Sanchez, C. On the nestmate-recognition system and territorial marking behaviour in the 523 antCamponotus rufipes. Insectes Sociaux 31, 302-315 (1984).

524 15. Loreto, R. G. et al. Foraging ants trade off further for faster: use of natural bridges and trunk trail 525 permanency in carpenter ants. Naturwissenschaften 100, 957-963 (2013).

526 16. Evans, H. C., Elliot, S. L. \& Hughes, D. P. Hidden Diversity Behind the Zombie-Ant Fungus

527 Ophiocordyceps unilateralis: Four New Species Described from Carpenter Ants in Minas Gerais,

$528 \quad$ Brazil. PLOS ONE 6, e17024 (2011).

529 17. Loreto, R. G., Elliot, S. L., Freitas, M. L. R., Pereira, T. M. \& Hughes, D. P. Long-Term Disease

530 Dynamics for a Specialized Parasite of Ant Societies: A Field Study. PLOS ONE 9, e103516 (2014).

531 18. Evans, H. C. \& Samson, R. A. Cordyceps species and their anamorphs pathogenic on ants

532 (Formicidae) in tropical forest ecosystems II. The Camponotus (Formicinae) complex. Trans. Br.

533 Mycol. Soc. 82, 127-150 (1984).

534 19. Araújo, J. \& Hughes, D. The fungal spore: myrmecophilous Ophiocordyceps as a case study. (CRC 535 Press, USA, 2017).

536 20. Del-Claro, K. \& Oliveira, P. S. Ant-Homoptera Interactions in a Neotropical Savanna: The

537 Honeydew-Producing Treehopper, Guayaquila xiphias (Membracidae), and its Associated Ant Fauna 538 on Didymopanax vinosum (Araliaceae) 1. Biotropica 31, 135-144 (1999).

539 21. Burd, M., Archer, D., Aranwela, N. \& Stradling, D. J. Traffic Dynamics of the Leaf-Cutting Ant, $540 \quad$ Atta cephalotes. Am. Nat. 159, 283-293 (2002).

541 22. Yang, L., Zhang, Y., Chen, J., Zhang, S. \& Chen, D. Z. Suggestive Annotation: A Deep Active

542 Learning Framework for Biomedical Image Segmentation. in Medical Image Computing and

543 Computer-Assisted Intervention - MICCAI 2017 399-407 (Springer, Cham, 2017). doi:10.1007/978-

544 3-319-66179-7_46 
23. He, K., Gkioxari, G., Dollár, P. \& Girshick, R. Mask R-CNN. in 2017 IEEE International Conference on Computer Vision (ICCV) 2980-2988 (2017). doi:10.1109/ICCV.2017.322

24. Chen, J., Harvey, C. W., Alber, M. S. \& Chen, D. Z. A Matching Model Based on Earth Mover's

548 Distance for Tracking Myxococcus Xanthus. in Medical Image Computing and Computer-Assisted

549 Intervention - MICCAI 2014 113-120 (Springer, Cham, 2014). doi:10.1007/978-3-319-10470-6_15

550 25. Chen, J., Alber, M. S. \& Chen, D. Z. A Hybrid Approach for Segmentation and Tracking of

551 Myxococcus Xanthus Swarms. IEEE Trans. Med. Imaging 35, 2074-2084 (2016).

552 26. R Core Team. R: A Language and Environment for Statistical Computing. (R Foundation for $553 \quad$ Statistical Computing, 2018).

554 27. RStudio Team. RStudio: Integrated Development Environment for R. (RStudio, Inc., 2016).

555 28. Benhamou, S. How to reliably estimate the tortuosity of an animal's path:: straightness, sinuosity, or fractal dimension? J. Theor. Biol. 229, 209-220 (2004).

29. Almeida, P. J. A. L., Vieira, M. V., Kajin, M., Forero-Medina, G. \& Cerqueira, R. Indices of movement behaviour: conceptual background, effects of scale and location errors. Zoologia 27, (2010).

30. Wilensky, U. NetLogo. (Center for Connected Learning and Computer-Based Modeling, 1999).

31. Bates, D., Mächler, M., Bolker, B. \& Walker, S. Fitting Linear Mixed-Effects Models Using lme4. J. Stat. Softw. 67, 1-48 (2015).

32. Kuznetsova, A., Brockhoff, P. B. \& Christensen, R. H. B. 1merTest Package: Tests in Linear Mixed Effects Models. J. Stat. Softw. 82, (2017).

33. Greene, M. J. \& Gordon, D. M. Social insects: Cuticular hydrocarbons inform task decisions. Nature 423, 32 (2003).

34. Heller, N. E. \& Gordon, D. M. Seasonal spatial dynamics and causes of nest movement in colonies of the invasive Argentine ant (Linepithema humile). Ecol. Entomol. (2006). molecules? Phys. Life Rev. 3, 162-187 (2006). 
36. Gordon, D. M. Ant encounters: interaction networks and colony behavior. (Princeton University Press, 2010).

573 37. Übler, E., Kern, F., Bestmann, H. J., Hölldobler, B. \& Attygalle, A. B. Trail pheromone of two

574 formicine ants,Camponotus silvicola and C. rufipes (Hymenoptera: Formicidae).

$575 \quad$ Naturwissenschaften 82, 523-525 (1995).

576 38. Goss, S., Aron, S., Deneubourg, J. L. \& Pasteels, J. M. Self-organized shortcuts in the Argentine ant. $577 \quad$ Naturwissenschaften 76, 579-581 (1989).

578 39. Beckers, R., Deneubourg, J. L. \& Goss, S. Trail laying behaviour during food recruitment in the ant 579 Lasius niger (L.). Insectes Sociaux 39, 59-72 (1992).

580 40. Vittori, K. et al. Path efficiency of ant foraging trails in an artificial network. J. Theor. Biol. 239, $507-515$ (2006).

41. Burd, M., Shiwakoti, N., Sarvi, M. \& Rose, G. Nest architecture and traffic flow: large potential effects from small structural features. Ecol. Entomol. 35, 464-468 (2010).

42. Bernadou, A. \& Fourcassié, V. Does substrate coarseness matter for foraging ants? An experiment with Lasius niger (Hymenoptera; Formicidae). J. Insect Physiol. 54, 534-542 (2008).

43. Yates, A. A. \& Nonacs, P. Preference for straight-line paths in recruitment trail formation of the Argentine ant, Linepithema humile. Insectes Sociaux 63, 501-505 (2016).

44. Pearce-Duvet, J. M. C., Elemans, C. P. H. \& Feener, D. H. Walking the line: search behavior and foraging success in ant species. Behav. Ecol. 22, 501-509 (2011).

45. Adler, F. R. \& Gordon, D. M. Information Collection and Spread by Networks of Patrolling Ants. Am. Nat. 140, 373-400 (1992).

46. Gordon, D. M. Behavioral Flexibility and the Foraging Ecology of Seed-Eating Ants. Am. Nat. 138, 379-411 (1991).

47. Evison, S. E. F., Hart, A. G. \& Jackson, D. E. Minor workers have a major role in the maintenance of leafcutter ant pheromone trails. Anim. Behav. 75, 963-969 (2008). 
48. Feener, D. H. \& Moss, K. A. G. Defense against parasites by hitchhikers in leaf-cutting ants: a quantitative assessment. Behav. Ecol. Sociobiol. 26, 17-29 (1990).

49. Spaethe, J., Brockmann, A., Halbig, C. \& Tautz, J. Size determines antennal sensitivity and behavioral threshold to odors in bumblebee workers. Naturwissenschaften 94, 733-739 (2007).

50. Detrain, C. \& Pasteels, J. M. Caste differences in behavioral thresholds as a basis for polyethism during food recruitment in the ant, Pheidole pallidula (Nyl.) (Hymenoptera: Myrmicinae). J. Insect Behav. 4, 157-176 (1991).

51. Masuda, N., O’shea-Wheller, T. A., Doran, C. \& Franks, N. R. Computational model of collective nest selection by ants with heterogeneous acceptance thresholds. R. Soc. Open Sci. 2, 140533 (2015).

52. Latty, T., Holmes, M. J., Makinson, J. C. \& Beekman, M. Argentine ants (Linepithema humile) use adaptable transportation networks to track changes in resource quality. J. Exp. Biol. 220, 686-694 (2017).

53. Campos, D., Bartumeus, F., Méndez, V., Andrade, J. S. \& Espadaler, X. Variability in individual activity bursts improves ant foraging success. J. R. Soc. Interface 13, 20160856 (2016).

54. Von Frisch, K. The dance language and orientation of bees. (1967).

55. Seeley, T. D. Division of labor between scouts and recruits in honeybee foraging. Behav. Ecol. Sociobiol. 12, 253-259 (1983).

56. Howard, J. J., Henneman, L. M., Cronin, G., Fox, J. A. \& Hormiga, G. Conditioning of scouts and recruits during foraging by a leaf-cutting ant,Atta colombica. Anim. Behav. 52, 299-306 (1996).

57. Crawford, D. L. \& Rissing, S. W. Regulation of recruitment by individual scouts inFormica oreas Wheeler (Hymenoptera, Formicidae). Insectes Sociaux 30, 177-183 (1983).

58. Porter, S. D. \& Jorgensen, C. D. Foragers of the harvester ant, Pogonomyrmex owyheei: a disposable caste? Behav. Ecol. Sociobiol. 9, 247-256 (1981).

59. Ingram, K. K., Pilko, A., Heer, J. \& Gordon, D. M. Colony life history and lifetime reproductive success of red harvester ant colonies. J. Anim. Ecol. 82, 540-550 (2013). 
622 Acknowledgements. We thank the Department of Forest Engineering at the Federal University of Viçosa

623 for allowing us to perform this study at the Research Station of Mata do Pariso and Dr. Simon Elliot for

624 hosting us in his laboratory. We are grateful to Charissa de Bekker who helped capture the behavior in

625 Video S11. This work was supported in part by National Science Foundation Grants IOS-1558062 and

626 EEID 1414296 to D.P.H, NSF CCF-1617735 to D.Z.C., and NIH Grant R01 GM116927-02 to D.P.H. and

627 D.Z.C.

628

629 Author Contributions Statement. D.P.H., R.G.L, and N.I. conceived and designed the study. N.I. and

630 C.K. performed the field work with technical input from R.GL.. Y.Z. and D.Z.C. created the computer

631 model and processed the data. N.I. analyzed the data and wrote the manuscript with guidance from

632 D.P.H.. All authors reviewed the manuscript.

633

634 Additional Information. Competing Interests: The authors declare no competing interests. 